\title{
Short communication: Camel milk ameliorates inflammatory responses and oxidative stress and downregulates mitogen-activated protein kinase signaling pathways in lipopolysaccharide-induced acute respiratory distress syndrome in rats
}

\author{
Wei-Wei Zhu, ${ }^{* 1}$ Gui-Qing Kong, ${ }^{* 1}$ Ming-Ming Ma, $\dagger^{1}$ Yan Li, $\ddagger^{1}$ Xiao Huang, ${ }^{*}$ Li-Peng Wang, ${ }^{*}$ Zhen-Yi Peng, $§$ \\ Xiao-Hua Zhang,\# Xiang-Yong Liu, $\|^{2}$ and Xiao-Zhi Wang ${ }^{* 2}$ \\ *Department of Intensive Care Unit, Affiliated Hospital of Binzhou Medical University, Binzhou, 256603, Shandong Province, China \\ †Department of Intensive Care Unit, Hulunbeier People's Hospital, Hulunbeier, 021000, Inner Mongolia Autonomous Region, China \\ tDepartment of Respiratory Medicine, and \\ $\S$ Department of Ultrasound, Affiliated Hospital of Binzhou Medical University, Binzhou, 256603, Shandong Province, China \\ \#Department of Biotechnology, and \\ IIDepartment of Cell Biology, Binzhou Medical University, Yantai, 264003, Shandong Province, China
}

\begin{abstract}
Acute respiratory distress syndrome (ARDS) is a complex syndrome disorder with high mortality rate. Camel milk (CM) contains antiinflammatory and antioxidant properties and protects against numerous diseases. This study aimed to demonstrate the function of CM in lipopolysaccharide (LPS)-induced ARDS in rats. Camel milk reduced the lung wet:dry weight ratio and significantly reduced LPS-induced increases in neutrophil infiltration, interstitial and intra-alveolar edema, thickness of the alveolar wall, and lung injury scores of lung tissues. It also had antiinflammatory and antioxidant effects on LPS-induced ARDS. After LPS stimulation, the levels of proinflammatory cytokines (tumor necrosis factor- $\alpha$, IL-10, and IL-1 $\beta$ ) in serum and oxidative stress markers (malondialdehyde, myeloperoxidase, and total antioxidant capacity) in lung tissue were notably attenuated by CM. Camel milk also downregulated mitogen-activated protein kinase signaling pathways. Given these results, CM is a potential complementary food for ARDS treatment.
\end{abstract}

Key words: acute respiratory distress syndrome (ARDS), camel milk (CM), inflammatory response, oxidative stress, mitogen-activated protein kinase (MAPK) signaling pathway

\section{Short Communication}

Acute respiratory distress syndrome (ARDS) is characterized by alveolar epithelial and endothelial bar-

Received June 24, 2015.

Accepted September 26, 2015.

${ }^{1}$ These authors contributed equally to this study.

${ }^{2}$ Corresponding authors: hxicuwxz@163.com and liuxiangyong81@ gmail.com rier disruption, significant inflammation, and severe respiratory failure, with a high mortality rate (Ashbaugh et al., 1967; Li et al., 2014). Several candidate therapy methods, such as glucocorticoid, ulinastatin, and fluid management, have been used for ARDS treatment (Ware and Matthay, 2000; Diaz et al., 2010; Zhang et al., 2013). However, the mortality caused by ARDS remains high and developing novel therapy methods for ARDS is necessary. Camel milk (CM) possesses protective and ameliorating properties against alcohol-induced hepatic injury (Darwish et al., 2012), 2,4,6-trinitrobenzenesulfonic acid (TNBS)-induced colitis (Arab et al., 2014), autism spectrum disorder (Al-Ayadhi and Elamin, 2013), and type 1 diabetes (Agrawal et al., 2005). The CM used in this study was purchased from Wang Yuan Camel Milk Company (Xinjiang, China); it was obtained from Bactrian camels living in the desert area of Altai of Xinjiang Uyghur Autonomous Region in China. Camel milk contains higher contents of minerals and vitamins $\mathrm{A}, \mathrm{B}_{2}, \mathrm{E}$, and $\mathrm{C}$ than cow milk (Zhang et al., 2005; Konuspayeva et al., 2007); it is also abundant in lactoferrin, a protective protein with antioxidant and antiinflammatory properties (Håversen et al., 2002; Legrand et al., 2005; Konuspayeva et al., 2007).

This study aimed to determine the potential antiinflammatory and antioxidant properties of $\mathrm{CM}$ and the regulation of mitogen-activated protein kinase (MAPK) signaling pathways in LPS-induced ARDS in rats.

Male Wistar rats weighing 150 to $200 \mathrm{~g}$ were purchased from Lu Kang Animal Feed Sales Center (Shandong, China) and used in compliance with the Guide for the Care and Use of Laboratory Animals (National Research Council, 1996). Rats were randomly classified into 4 groups: control, CM, LPS, and LPS+CM. The LPS+CM and CM groups were intragastrically admin- 
A
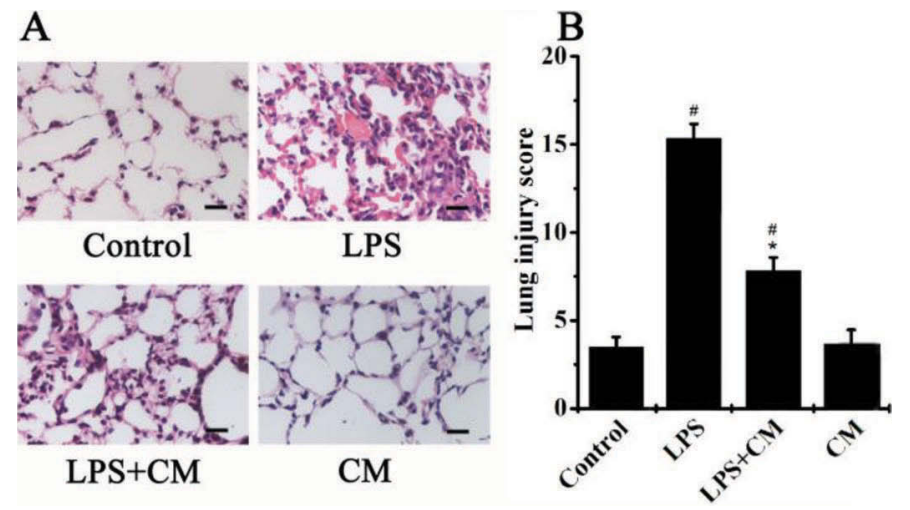

Figure 1. Camel milk (CM) ameliorates pulmonary pathological change in LPS-induced acute respiratory distress syndrome in rats. A representative view $(400 \times$ magnification) from each group is demonstrated. Values shown in the graphs are mean $\pm \mathrm{SD}(\mathrm{n}=12$ rats per group) of 3 independent experiments. \#P<0.05 compared with the control group; ${ }^{*} P<0.05$ compared with the LPS group. Scale bar $=$ $20 \mu \mathrm{m}$. Color version available online.

istered with $\mathrm{CM}(10 \mathrm{~mL} / \mathrm{kg}$ of $\mathrm{BW})$ twice a day for 2 wk (Arab et al., 2014). Rats from the control and LPS groups received an equal volume of saline instead of CM. After $14 \mathrm{~d}, 10 \mathrm{mg} / \mathrm{kg}$ LPS was dissolved in $100 \mu \mathrm{L}$ of PBS and intraperitoneally (i.p.) injected into rats to induce ARDS. The control and CM rats were given 100 $\mu \mathrm{L}$ of PBS without LPS. All rats were killed after $6 \mathrm{~h}$ of LPS treatment.

The results are presented as mean \pm standard deviation (SD). The statistical significance of the results was analyzed by one-way ANOVA and the subsequent Bonferroni post hoc test. The $P$-value was two-tailed and considered statistically significant if $<0.05$.
After hematoxylin and eosin staining, pulmonary histopathological examination was conducted by observing the slides under a light microscope (400× magnification; Li et al., 2014). Lung injury score was assessed by evaluating alveolar congestion, hemorrhage, infiltration, or aggregation of neutrophils in the airspace or vessel wall and the thickness of the alveolar wall or hyaline membrane (Nishina et al., 1997). The severity of lung injury was scored as follows: $0=$ minimum; 1 = mild; $2=$ moderate; $3=$ severe; and $4=$ maximum. Histological alterations were not observed in the control or CM groups. Camel milk remitted pulmonary histopathological change induced by ARDS in rats compared with the LPS group (Figure 1A). As shown in Figure 1A, lung sections obtained from the control and CM groups presented a normal structure. In contrast, the LPS groups showed extensive morphological damage, such as areas of neutrophil infiltration, thickening of the alveolar wall, as well as interstitial and intra-alveolar edema. However, LPS-induced pathological changes were significantly attenuated by CM. Six high-magnification fields were randomly selected and graded to obtain the average lung injury score for each stained sample (Figure 1B). The LIS in the LPS group was significantly higher than that in the control and CM groups. However, CM obviously inhibited LIS compared with that in the LPS group.

The severity of pulmonary edema was evaluated by calculating the wet:dry weight ratio. No differences existed between the control and CM groups. Lipopolysaccharide caused a significant increase in lung wet:dry weight ratio compared with the control group, and CM reduced the pulmonary edema compared with the LPS group (Figure 2A).
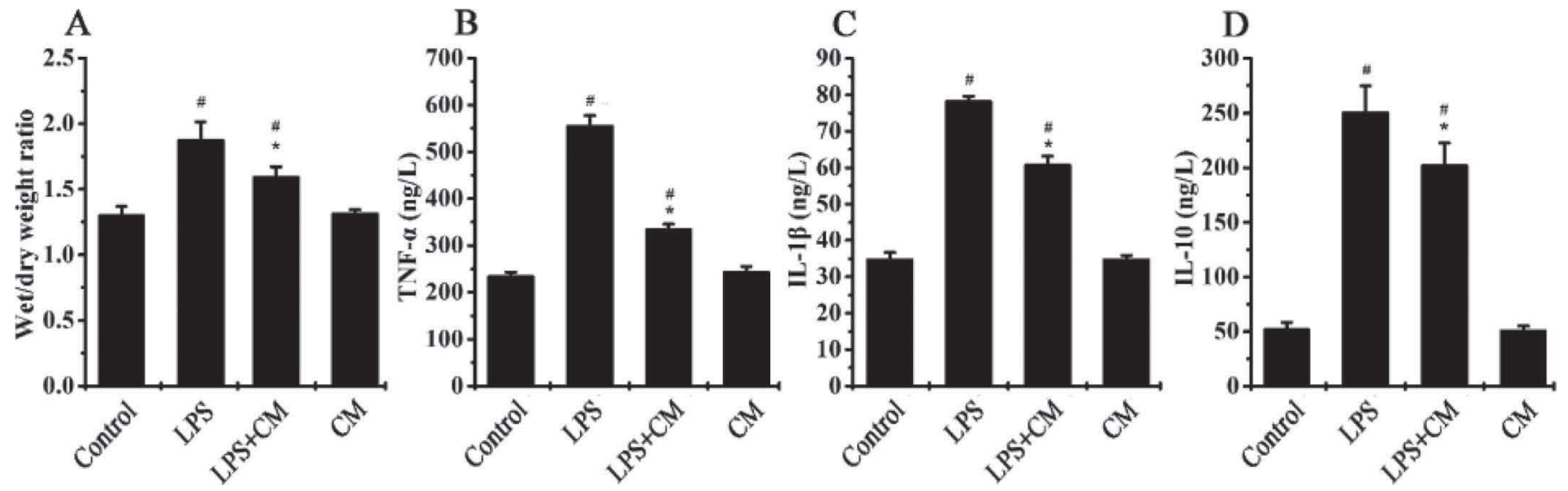

Figure 2. Camel milk $(\mathrm{CM})$ relieves pulmonary edema and inflammatory responses in LPS-induced acute respiratory distress syndrome in rats. (A) Lung wet/dry weight ratio; (B-D) serum proinflammatory cytokine levels of tumor necrosis factor (TNF)- $\alpha$, IL-1 $\beta$, and IL-10, respectively (determined using specific ELISA kits). Values shown are means $\pm \mathrm{SD}(\mathrm{n}=12$ rats per group) of 3 independent experiments. $\# P<0.05$ compared with the control group; ${ }^{*} P<0.05$ compared with the LPS group. 
Proinflammatory cytokines, such as tumor necrosis factor (TNF) $\alpha$, IL-1 $\beta$, and IL-10, play an important role in the pathogenesis of ARDS (Bustos et al., 2013; Ma et al., 2015). Total antioxidant capacity (TAOC), a marker of antioxidant defense, depicts the synergistic interaction among different antioxidants (Arab et al., 2014). We assessed malondialdehyde (MDA), which can form adducts with cellular proteins and nucleic acids (Karapetsa et al., 2013), and myeloperoxidase (MPO) activity, which is a marker of neutrophil influx into tissues, to quantify neutrophil accumulation in tissues. Levels of proinflammatory cytokines (TNF- $\alpha$, IL-10, and IL-1 $\beta$ ) in serum and oxidative stress markers (MDA, MPO, and TAOC) in lung tissue were detected using ELISA kits. The results indicated the efficacy of $\mathrm{CM}$ in relieving inflammatory responses and oxidative stress in LPS-induced ARDS in rats (Figures 2 and 3). After LPS stimulation, the levels of TNF- $\alpha$, IL-10, and IL-1 $\beta$ in serum and the oxidative stress markers MDA, MPO, and TAOC in lung tissue were significantly higher compared with that in the control group. Furthermore, treatment with CM decreased the levels of TNF- $\alpha$, IL-10, IL-1 $\beta$, MDA, TAOC, and MPO compared with those in the LPS group. In contrast,

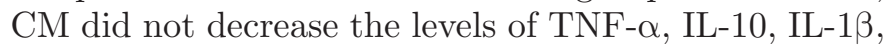
MDA, TAOC, or MPO compared with those in the LPS group.

The MAPK signaling pathway includes 3 main signaling cascades, including JNK (c-Jun N-terminal kinase), ERK (extracellular signal-regulated kinases), and p38 kinase (Rao et al., 2001). Activation of the MAPK signaling pathway in turn activates transcription regulators of inflammation in the nucleus, which plays an important role in numerous cellular physiologies, such as proliferation, differentiation, and apoptosis (Bost et al., 2005; Ma et al., 2015). Thus, the effects

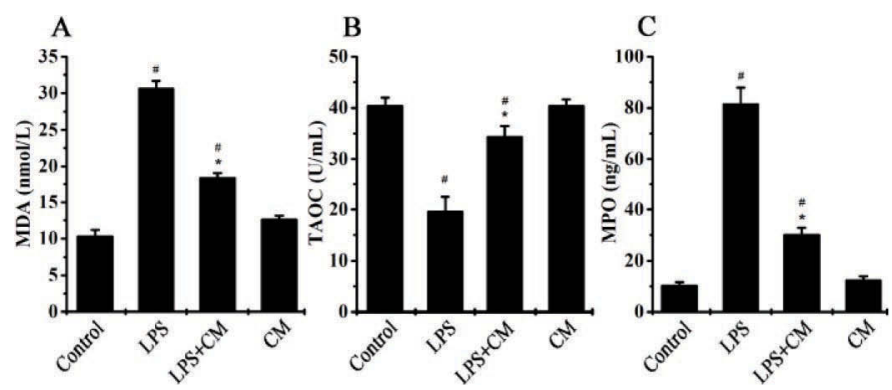

Figure 3. Camel milk (CM) alleviates oxidative stress in LPSinduced acute respiratory distress syndrome in rats. Levels of malondialdehyde (MDA; A), total antioxidant capacity (TAOC; B), and myeloperoxidase (MPO; C) in lung were tested using ELISA kits. Values shown are means $\pm \mathrm{SD}(\mathrm{n}=12$ rats per group $)$ of 3 independent experiments. $\# P<0.05$ compared with the control group; ${ }^{*} P<0.05$ compared with the LPS group.
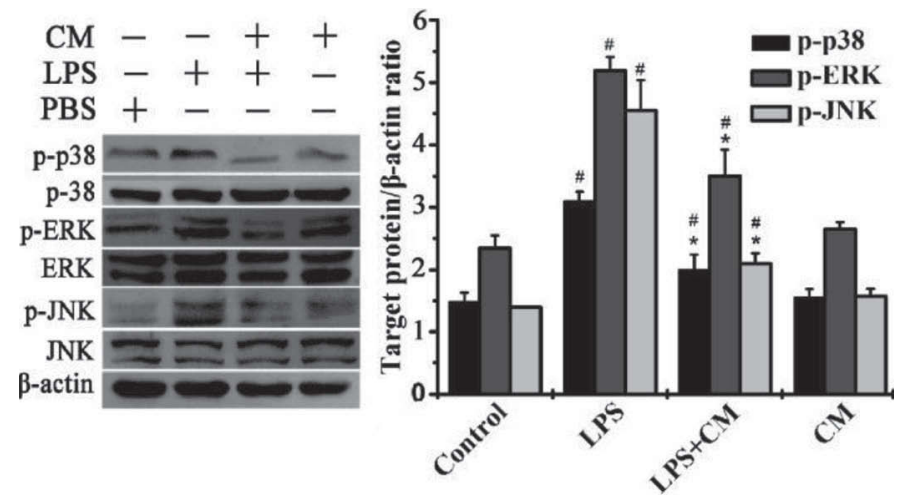

Figure 4. Camel milk (CM) downregulates mitogen-activated protein kinases (MAPK) signaling pathways in LPS-induced acute respiratory distress syndrome in rats. Cellular proteins (obtained from the lungs) of phosphorylated (p) or complete forms of 3 MAPK molecules (ERK, p38, and JNK); PBS $=$ control. Values shown are means \pm $\mathrm{SD}(\mathrm{n}=12$ rats per group) of 3 independent experiments. $\# P<0.05$ compared with the control group; ${ }^{*} P<0.05$ compared with the LPS group.

of $\mathrm{CM}$ on the activation of phospho-ERK, phosphoJNK, and phospho-p38 were investigated. Western blot analysis results showed that LPS stimulation increased the phosphorylation levels of components of the MAPK signaling pathway and was significantly inhibited by CM (Figure 4).

In conclusion, CM ameliorates inflammatory responses and oxidative stress and downregulates MAPK signaling pathways in LPS-induced ARDS in rats. The use of CM in combination with other drugs may be considered in the therapy of LPS-induced ARDS.

\section{ACKNOWLEDGMENTS}

This work received funding from the Natural Science Foundation of Shandong Province, China (ZR2014HM112, ZR2014HL004, UF201403), the Science and Technology Development Plan of Shandong Province, China (2011GSF11830), and Taishan Scholar project of Shandong Province, China.

\section{REFERENCES}

Agrawal, R. P., R. Beniwal, D. K. Kochar, F. C. Tuteja, S. K. Ghorui, M. S. Sahani, and S. Sharma. 2005. Camel milk as an adjunct to insulin therapy improves long-term glycemic control and reduction in doses of insulin in patients with type-1 diabetes: A 1 year randomized controlled trial. Diabetes Res. Clin. Pract. 68:176-177.

Al-Ayadhi, L. Y., and N. E. Elamin. 2013. Camel milk as a potential therapy as an antioxidant in autism spectrum disorder (ASD). Evid. Based Complement. Alternat. Med. 2013:602834.

Arab, H. H., S. A. Salama, A. H. Eid, H. A. Omar, E. A. Arafa, and I. A. Maghrabi., 2014. Camel's milk ameliorates TNBS-induced colitis in rats via downregulation of inflammatory cytokines and oxidative stress. Food Chem. Toxicol. 69:294-302.

Ashbaugh, D. G., D. B. Bigelow, T. L. Petty, and B. E. Levine. 1967. Acute respiratory distress in adults. Lancet 2:319-323. 
Bost, F., M. Aouadi, L. Caron, and B. Binetruy. 2005. The role of MAPKs in adipocyte differentiation and obesity. Biochimie 87:5156.

Bustos, M. L., L. Huleihel, E. M. Meyer, A. D. Donnenberg, V. S. Donnenberg, J. D. Sciurba, L. Mroz, B. J. McVerry, B. M. Ellis, N. Kaminski, and M. Rojas. 2013. Activation of human mesenchymal stem cells impacts their therapeutic abilities in lung injury by increasing interleukin (IL)-10 and IL-1RN levels. Stem Cells Transl. Med. 2:884-895.

Darwish, H. A., N. R. Abd Raboh, and A. Mahdy. 2012. Camel's milk alleviates alcohol-induced liver injury in rats. Food Chem. Toxicol. 50:1377-1383.

Diaz, J. V., R. Brower, C. S. Calfee, and M. A. Matthay. 2010. Therapeutic strategies for severe acute lung injury. Crit. Care Med. 38:1644-1650.

Håversen, L., B. G. Ohlsson, M. Hahn-Zoric, L. A. Hanson, and I. Mattsby-Baltzer. 2002. Lactoferrin down-regulates the LPS-induced cytokine production in monocytic cells via NF-kappa B. Cell. Immunol. 220:83-95.

Karapetsa, M., M. Pitsika, N. Goutzourelas, D. Stagos, A. Tousia Becker, and E. Zakynthinos. 2013. Oxidative status in ICU patients with septic shock. Food Chem. Toxicol. 61:106-111.

Konuspayeva, G., B. Faye, G. Loiseau, and D. Levieux. 2007. Lactoferrin and immunoglobulin contents in camel's milk (Camelus bactrianus, Camelus dromedarius, and hybrids) from Kazakhstan. J. Dairy Sci. 90:38-46.

Legrand, D., E. Elass, M. Carpentier, and J. Mazurier. 2005. Lactoferrin: A modulator of immune and inflammatory responses. Cell. Mol. Life Sci. 62:2549-2559.
Li, Y., X. Y. Liu, M. M. Ma, Z. J. Qi, X. Q. Zhang, Z. Li, G. H. Cao, J. Li, W. W. Zhu, and X. Z. Wang. 2014. Changes in intestinal microflora in rats with acute respiratory distress syndrome. World J. Gastroenterol. 20:5849-5858.

Ma, M. M., Y. Li, X. Y. Liu, W. W. Zhu, X. Ren, G. Q. Kong, X. Huang, L. P. Wang, L. Q. Luo, and X. Z. Wang. 2015. Cyanidin3-O-glucoside ameliorates lipopolysaccharide-induced injury both in vivo and in vitro suppression of NF- $\mathrm{KB}$ and MAPK pathways. Inflammation 38:1669-1682.

National Research Council. 1996. Guide for the Care and Use of Laboratory Animals. Institute of Laboratory Animal Resources, National Research Council, Washington, DC.

Nishina, K., K. Mikawa, Y. Takao, N. Maekawa, M. Shiga, and H. Obara. 1997. ONO-5046, an elastase inhibitor, attenuates endotoxin-induced acute lung injury in rabbits. Anesth. Analg. 84:10971103.

Rao, K. M. 2001. MAP kinase activation in macrophages. J. Leukoc. Biol. 69:3-10.

Ware, L. B., and M. A. Matthay. 2000. The acute respiratory distress syndrome. N. Engl. J. Med. 342:1334-1349.

Zhang, H., J. Yao, D. Zhao, H. Liu, J. Li, and M. Guo. 2005. Changes in chemical composition of Alxa bactrian camel milk during lactation. J. Dairy Sci. 88:3402-3410.

Zhang, X. Q., C. J. Lv, X. Y. Liu, D. Hao, J. Qin, H. H. Tian, Y. Li, and X. Z. Wang. 2013. Genome-wide analysis of DNA methylation in rat lungs with lipopolysaccharide-induced acute lung injury. Mol. Med. Rep. 7:1417-1424. 